dược sĩ có thể tư vấn bằng điện thoại qua ứng dụng hỗ trợ nên NB không cần phải chờ đợi lâu ở bệnh viện để được tư vấn mà có thể hỏi các thông tin về thuốc khi ở nhà. Nhiềunghiên cứu trên thế giới đã chứng minh sự can thiêp của nhân viên y tế tác động tích cực lên sự tuân thủ sử dụng thuốc của NB. Nghiên cứu ở Canada năm 2014 về tác động của dược sĩ trong việc quản lý NBTHA, nhóm can thiệp có tỷ lệ tuân thủ tăng cao hơn so với nhóm chứng (15\% vs $2,2 \%)$ (7). Một nghiên cứu ở Nepal phỏng vấn 332 NBTHA cho thấy nhóm có sự tư vấn của dược sĩ có tỷ lệ tuân thủ cao hơn nhóm chứng $(86,15 \%$ vs $13,86 \%$ ) (8). Chương trình CTHDSDT cũng ghi nhận tác động tích cực của Dược sĩ trong việc giúp tăng tỷ lệ NB tuân thủ sử dụng thuốc.

\section{KẾT LUẬN}

Chương trình can thiệp hướng dẫn sử dụng thuốc cho NBTHA tại Bệnh viện đa khoa Thống Nhất tỉnh Đồng Nai bước đầu đạt được một số hiệu quả nhất định, tạo tiền đề cho việc tiếp tục triển khai và áp dụng chương trình này tại Bệnh viện.

\section{TÀI LIÊU THAM KHẢO}

1. Thái Khoa Bảo Châu (2016). Nghiên cứu tình hình sử dụng thuốc trong điều trị tăng huyết áp tại Bệnh viện trường đại học Y Dược Huế. Tạp chí Y Dược Huế số 32, trang 76-84.

2. Nguyễn Thị Thủy (2018). Khảo sát kiến thức về bênhh tăng huyết áp của bệnh nhân tăng huyết áp điêuu tri nội trú tai bênh viện quân y 103 năm 2017. Tạp chí Y Dược học quân sự, số 1, năm 2018, trang 29-35.

3. Ashp (1997). Medication Therapy and Patient Care: Organization and Delivery of ServicesGuidelines ASHP Guidelines on PharmacistConducted Patient Education and Counseling. American Journal of Health-System Pharmacy, vol. 54, No. 4. pp. 340-342.

4. Emilio Márquez-Contreras (2006). Efficacy of a Home Blood Pressure Monitoring Programme on Therapeutic Compliance in Hypertension: The EAPACUM-HTA Study. Journal of hypertension, Vol 24, pp 169-175.

5. Jean-Pierre Fina Lubaki (2009). Reasons for noncompliance among patients with hypertension at Vanga Hospital, Bandundu Province, Democratic Republic of Congo: A qualitative study. African Journal of Primary Health Care \& Family Medicine, Vol 1, pp 1-5

6. Morisky D.E., et al. (2008). Predictive validity of a medication adherence measure in an outpatient setting. The Journal of Clinical Hypertension, 10 (5), pp. 348-354.

7. Pharmacists Association and GreenShield Canada (2014). Impact of Community Pharmacist Interventions in Hypertension Management on Patient Outcomes: A Randomized Controlled Trial Final Project Report. British Journal of Clinical Pharmacology, 78 (6), pp 1238-1247.

8. Sumitra Shrestha (2019). Impact of Pharmacist Counselling on Medication Adherence among Elderly Patients on Antihypertensive Therapy in a Tertiary Care Hospital of Nepal.Europasian Journal of medical Sciences, Vol 1, Jul-Dec 2019.

\title{
NGHIÊN CỨU BÀO CHẾ VIÊN NANG CHỨA ESOMEPRAZOL 20MG DẠNG HẠT BAO TAN TRONG RUỘT
}

\section{TÓM TẮT}

Muc tiêu: Bào chế viên nang chứa esomeprazol dạng hạt bao tan trong ruột. Đối tượng và phương pháp: Đối tượng nghiên cứu là esomeprazol với polymer Eudragit ${ }^{\circledR}$ L30D-55. Khảo sát lớp bao mang hoạt chất (tỷ lệ cồn: nước, khối lượng PEG 6000, khối lượing talc, hàm lượng chất khô), khảo sát lớp bao cách ly (tỷ lê cồn: nước, khối lượng PEG 6000, hàm lượng chất khô), khảo sát lớp bao tan trong ruột (khối lượng $\mathrm{TiO}_{2}$, độ tăng trọng lớp bao tan trong ruột) đến quá trình tạo hạt nhằm xác định độ hoà tan của hạt trong môi trường acid $\mathrm{HCl} 0,1 \mathrm{~N}$ trong 2 giờ là không quá $10 \%$ và trong môi trường đệm phosphat $\mathrm{pH} 6,8$

*Đai hoc Y Dước Cần Thơ

Chịu trách nhiệm chính: Nguyễn Thị Linh Tuyền

Email: ntltuyen@ctump.edu.vn

Ngày nhận bài: 1/3/2021

Ngày phản biên khoa hoc: 28/3/2021

Ngày duyệt bài: 25/4/2021

\section{Nguyễn Thị Linh Tuyền*, Phan Lê Hoài Ân*}

trong 30 phút không nhỏ hơn $80 \%$. Kết quả: ở lớp bao mang hoạt chất có tỷ lệ cồn: nhước là $3: 1$, khối lượng PEG 6000 là 2,1g, khổi lượng talc là 2,1g, hàm lượng chất khô là $8 \%$. Ơ lớp bao cách ly có tỷ lệ cồn: nước là 3:1, khối lượng PEG 6000 là 1,2g, hàm lượng chất khô là $6 \%$. Ở lớp bao tan trong ruột có khối lượng $\mathrm{TiO}_{2}$ là $1 \mathrm{~g}$, độ tăng trọng của lớp bao tan trong ruột là $30 \%$. Kết luân: đã bào chếviên nang chứa esomeprazol dạng hạt bao tan trong ruột đạt tiêu chuẩn của USP43.

Tư khoá: Esomeprazol, Eudragit ${ }^{\circledR}$ L30D-55, hạt.

\section{SUMMARY}

\section{FORMULATION OF ESOMEPRAZOLE 2OMG DELAYED RELEASE PELLETS CONTAINED CAPSULES}

Objectives: Preparation of capsules containing esomeprazole delayed release pellet. Materials and methods: The materials were esomeprazole with polymer Eudragit ${ }^{\circledR}$ L30D-55. Survey the active coating layer (alcohol: water ratio, PEG 6000 weight, talc 
weight, dry matter content), survey the insulation layer (alcohol: water ratio, PEG 6000 weight, dry matter content), survey the soluble layer in the intestine $\left(\mathrm{TiO}_{2}\right.$ weight, the weight gain of the intestinal layer) to the pellet process to determine the solubility in hydrochloric acid $0,1 \mathrm{~N}$ in 2 hours was more than $10 \%$ and in phosphate buffer medium $\mathrm{pH}$ 6.8 for 30 minutes not less than $80 \%$. Results: In the active coating layer, the alcohol: water ratio was 3:1, the weight of PEG 6000 was $2,1 \mathrm{~g}$, the weight of talc was $2,1 \mathrm{~g}$ and the dry matter content was $8 \%$. In the insulating layer, the alcohol: water ratio was $3: 1$, the weight of PEG 6000 was $1,2 \mathrm{~g}$ and the dry matter content was $6 \%$.In the intestinal soluble coating with $\mathrm{TiO}_{2}$ weight of $1 \mathrm{~g}$, the weight gain of the intestinal soluble coating was $30 \%$. Conclusion: Has prepared capsules containing esomeprazole delayed release pellets form to meet the standards of USP43.

Keywords: Esomeprazole, Eudragit ${ }^{\circledR}$ L30D-55, pellets.

\section{I. ĐĂT VẤN ĐỀ}

Esomeprazol là thuốc ức chế bơm proton được sử dụng rộng rãi nhất trong các phác đồ điêu trị viêm loét dạ dày tá tràng mang lại hiệu quả cao.

Esomeprazol là chất kém tan trong nước và kém ổn định dưới tác động của ánh sáng, nhiệt độ, độ ẩm, đặc biệt là trong môi trường acid dạ dày ${ }^{[1]}$, đây chính là vấn đề gây trở ngại lớn nhất cho các nhà bào chế. Chínhvilý do trên "Nghiên cứu bào chế viên nang chứa esomeprazol $20 \mathrm{mg}$ dạng hạt bao tan trong ruột" được đề xuất để khắc phục những nhược điểm trên và làm cho chế phẩm có độ ổn định $\mathrm{caO}^{[3]}$. Vì vậy nghiên cứu được tiến hành với mục tiêu bào chế viên nang chứa esomeprazol dạng hạt bao tan trong ruột đạt tiêu chuẩn kiểm nghiệm theo USP43.

\section{II. ĐỐI TƯỢNG VÀ PHƯƠNG PHÁP NGHIÊN CỨU \\ 2.1. Đối tượng nghiên cứu}

Nguyên liệu. Nguyên liệu chính sử dụng trong nghiên cứu là esomeprazol đạt tiêu chuẩn USP43 và Eudragit ${ }^{\circledR}$ L30D-55 đạt tiểu chuẩn của nhà sản xuất.

Thiết bị nghiên cứu. Máy sấy tầng sôi (Mini Glatt), máy đo tốc độ chảy (Erweka GTL), máy đo độ hòa tan (Pharmatest), máy đo tỳ trọng biểu kiến (Pharmatest).

\subsection{Phương pháp nghiên cứu}

Quy trình bào chế. Quy trình bào chế viên nang esomeprazol $20 \mathrm{mg}$ dạng hạt bao tan trong ruột trải qua các quá trình được tóm tắt ở sơ đồ Hình 1.

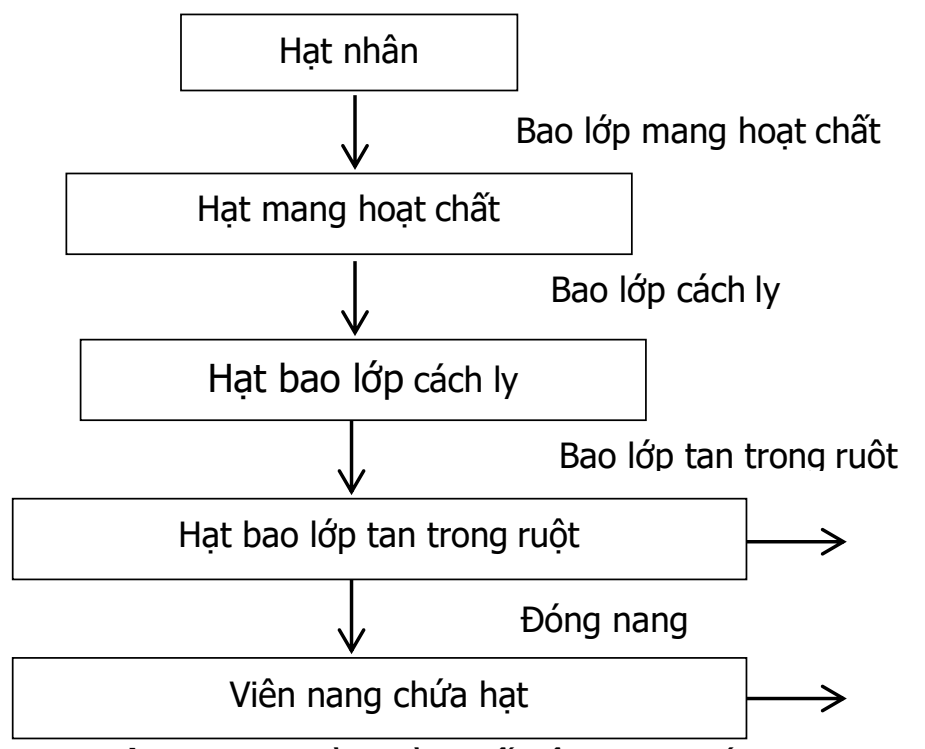

Kiểm nghiệm bán thành phẩm
Kiểm nghiệm thành phẩm

Hình 1. Quy trinh bào chế viên nang chứa esomeprazol 20mg dạng hạt bao tan trong ruột

Sau mỗi giai đoạn bao khảo sát lớp mang hoạt chất, lớp cách ly và lớp bao tan trong ruột, tiến hành tính hiệu suất bao và kiểm tra độ hoà tan của hạt trong môi trường đệm phosphat $\mathrm{pH}$ 6,8 như sau:

Xác định hiệu suất bao bằng công thức:

$$
H=\frac{m}{M} \cdot 100 \%
$$

Trong đó: H: hiệu suất bao hạt (\%)

$\mathrm{m}$ : khối lượng hạt tăng lên sau bao $(\mathrm{g})$

M: khối lượng chất khô trong dịch bao $(\mathrm{g})$

Xác định độ hoà tan trong môi trường acid $\mathrm{HCl} 0,1 \mathrm{~N}$

Thiết bị: cánh khuấy. Tốc độ quay: 100 vòng/phút. Môi trường hoà tan: $300 \mathrm{ml}$ dung dịch $\mathrm{HCl} 0,1 \mathrm{~N}$. Thời gian hoà tan: 2 giờ. Nhiệt độ môi 
trường hoà tan: $37 \pm 0,5^{\circ} \mathrm{C}$.

Mầu chuẩn: Dung dịch esomeprazol pha trong đệm phosphat pH 6,8 với nồng độ $20 \mu \mathrm{g} / \mathrm{ml}$.

Mẫu thư': Cân chính xác lượng hạt có chứa khoảng $20 \mathrm{mg}$ esomeprazol cho vào môi trường hoà tan. Sau 2 giờ, lọc toàn bộ dịch hoà tan qua rây $125 \mu \mathrm{m}$, giữ lại lượng hạt trên rây. Lấy lượng hat trên đem đi định lượng esomeprazol còn lại. Từ đó tính được lượng dược chất đã hoà tan trong aicd sau 2 giờ. Cho lượng hạt sau khi thử hoà tan trong môi trường aicd vào bình định mức $10 \mathrm{ml}$, thêm khoảng $7 \mathrm{ml} \mathrm{MeOH}$, lắc siêu âm khoảng 15 phút. Thêm $\mathrm{MeOH}$ vừa đủ đến vạch, lắc đều. Hút chính xác $1 \mathrm{ml}$ dung dịch này cho vào bình định mức $100 \mathrm{ml}$, thêm dung dịch đệm phosphat $\mathrm{pH} 6,8$ vừa đủ đến vach, lắc đều. Loc lấy dịch lọc, bỏ $20 \mathrm{ml}$ dịch lọc đầu. Đo độ hấp thu của dịch lọc tại bước sóng $301 \mathrm{~nm}$.

Mẫu trắng: Dung dịch đệm phosphat pH 6,8.

Phần trăm esomeprazol giải phóng ở thời điểm 2 giờ được tính theo công thức:

$$
\% \text { GPHC }=\left(1-\frac{D_{t} \cdot C_{c}}{m \cdot X \cdot D_{c}}\right) \cdot 100 \%
$$

Trong đó: \%GPHC: giải phóng hoạt chất (\%)

$\mathrm{X}$ : hàm lượng esomeprazol trong hạt

m: khối lượng hạt đem cân

$C_{c}$ : nồng độ dung dịch chuẩn $(\mu \mathrm{g} / \mathrm{ml})$

Dt: mật độ quang dung dịch thử

$D_{c}$ : mật độ quang dung dịch chuẩn

Xác định độ hoà tan trong môi trường đệm phosphat pH 6,8

Thiêt bi: cánh khuấy.Tốc độ quay: 100 vòng/phút. Môi trường hoà tan: $1000 \mathrm{ml}$ dung dịch đệm phosphat $\mathrm{pH} 6,8$. Thời gian hoà tan: 30 phút. Nhiệt độ môi trường hoà tan: $37 \pm 0,5^{\circ} \mathrm{C}$.

Mẫu chuẩn: dung dịch esomeprazol pha trong đệm phosphat pH 6,8 với nồng độ 20 g/ml.

Mấu thứ: cân chính xác lượng hạt có chứa khoảng $20 \mathrm{mg}$ esomeprazol cho vào môi trường đệm phosphat pH 6,8. Sau thời gian 30 phút, rút dịch loc đem đo quang ở bước sóng $\lambda_{\max }$.

Mẫu trắng: dung dịch đệm phosphat $\mathrm{pH} 6,8$.

Phần trăm esomeprazolgiải phóng ở thời điểm 2 giờ được tính theo công thức:

$$
\% G P H C=\frac{D_{t} \cdot C_{c}}{m \cdot X \cdot D_{c}} \cdot 100 \%
$$

Trong đó: \%GPHC: giải phóng hoạt chất (\%)

$X$ : hàm lượng esomeprazol trong hạt

m: khối lượng hạt đem cân

$C_{c}$ : nồng độ dung dịch chuẩn $(\mu \mathrm{g} / \mathrm{ml})$

Dt: mật độ quang dung dịch thử

$\mathrm{D}_{\mathrm{c}}$ : mật độ quang dung dịch chuẩn

\section{Khảo sát lớp bao mang hoạt chất}

Thành phần công thức dịch bao mang hoạt chất được trình bày ở bảng 1

Bảng 1. Thành phần lớp bao mang hoạt chât

\begin{tabular}{|c|c|c|}
\hline Thành phân & $\begin{array}{c}\text { Khối } \\
\text { lượng } \mathbf{( g )}\end{array}$ & Vai trò \\
\hline $\begin{array}{c}\text { Esomeprazol } \\
\text { magnesi }\end{array}$ & 3,5 & Hoạt chất \\
\hline HPMC E6 & 3,5 & Tá dược dính \\
\hline PEG 6000 & Khảo sát & Chất hoá dẻo \\
\hline MgO & 3,85 & Ôn định hoạt chất \\
\hline Talc & Khảo sát & Chống dính \\
\hline Tween 80 & 0,18 & Ôn định dịch bao \\
\hline Hô̂n hợp côn: nước & Khảo sát & Dung môi \\
\hline Pha chế dịch bao: hoà tan $\mathrm{HPMC} \mathrm{E6}, \mathrm{PEG}$
\end{tabular}
6000 và tween 80 vào hỗn hợp cồn: nước được (1). Cho từ từ bột talc, $\mathrm{MgO}$, esomeprazol vào (1), khuấy đều trền máy khuấy từ với tốc độ 300 vòng/phút đến khi đồng nhất, lọc qua rây $125 \mu \mathrm{m}$ thu được dịch bao mang hoạt chất. Dịch bao được khuây đều liên tục trong suốt quá trình bao để đảm bảo các chất rắn được phân bố đồng nhất trong hỗn dịch.

Mỗi mẻ bao sử dụng $30 \mathrm{~g}$ hạt trơ kích thước

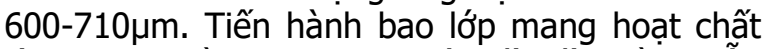
lên hạt trơ bằng phương pháp bồi dần bằng hỗn dịch trên máy sấy tầng sôi Mini Glatt với các thông số bao ở bảng 2.

Bảng 2. Thông số quá trinh bao lớp mang hoạt chất

\begin{tabular}{|c|c|}
\hline Thông số & Giá trị \\
\hline Tốc độ thối khí & $30-35 \mathrm{~m}^{3} / \mathrm{h}$ \\
\hline Nhiệt độ thối khí & $50^{\circ} \mathrm{C}$ \\
\hline Nhiệt độ mẻ & $42^{\circ} \mathrm{C}$ \\
\hline Đường kính ống dần & $1,5 \mathrm{~mm}$ \\
\hline Đường kính vòi phun & $0,7 \mathrm{~mm}$ \\
\hline Áp suất vòi phun & $1,1 \mathrm{bar}$ \\
\hline Tần suất xả lọc & 1 phút/lần \\
\hline Tốc độ phun dịch & $1,3 \mathrm{ml} /$ phút \\
\hline
\end{tabular}

Sau khi hết dịch tiếp tục sấy hạt ở $45^{\circ} \mathrm{C}$ trong 15 phút. Hạt sau khi sấy được lấy ra bảo quản ở lọ thuỷ tinh màu nâu, kín.

Khảo sát thành phần công thức dịch bao mang hoạt chất gồm:

$X_{1}$ : tỷ lệ cồn : nước $(1: 1,2: 1,3: 1,4: 1)$; $X_{1 T}$ : tỷ lệ cồn : nướctối ưu

$\dot{X}_{2}$ : khối lượng PEG 6000 (0,7g;1,4g; 2,1g;

2,8g; 3,5g); Х2т: khối lượng PEG 6000 tối ưu

$\mathrm{X}_{3}$ : khối lượng talc $(0,35 \mathrm{~g} ; 0,70 \mathrm{~g} ; 1,40 \mathrm{~g}$;

2,10g; 2,80g); Х Хт: khối lượng talc tối ưu

$\mathrm{X}_{4}$ : hàm lượng chất khô $(6 \%, 8 \%, 10 \%)$

Thành phần công thức dịch bao lớp mang hoạt chất được trình bày ở bảng 3 . 
Bảng 3. Thành phần công thức dịch bao lớp mang hoạt chất

\begin{tabular}{|c|c|c|c|c|c|c|c|c|c|}
\hline CT & $\mathbf{X 1}$ & $\mathbf{X 2}$ & $\mathbf{X 3}$ & $\mathbf{X 4}$ & $\mathbf{C T}$ & $\mathbf{X 1}$ & $\mathbf{X 2}$ & $\mathbf{X 3}$ & $\mathbf{X 4}$ \\
\hline CT1 & $1: 1$ & 2,10 & 1,40 & 8 & CT8 & $\mathrm{X}_{1 \mathrm{~T}}$ & 3,50 & 1,40 & 8 \\
\hline CT2 & $2: 1$ & 2,10 & 1,40 & 8 & CT9 & $\mathrm{X}_{1 \mathrm{~T}}$ & $\mathrm{X}_{2 \mathrm{~T}}$ & 0,35 & 8 \\
\hline CT3 & $3: 1$ & 2,10 & 1,40 & 8 & $\mathbf{C T 1 0}$ & $\mathrm{X}_{1 \mathrm{~T}}$ & $\mathrm{X}_{2 \mathrm{~T}}$ & 0,70 & 8 \\
\hline CT4 & $4: 1$ & 2,10 & 1,40 & 8 & $\mathbf{C T 1 1}$ & $\mathrm{X}_{1 \mathrm{~T}}$ & $\mathrm{X}_{2 \mathrm{~T}}$ & 2,10 & 8 \\
\hline CT5 & $\mathrm{X}_{1 \mathrm{~T}}$ & 0,70 & 1,40 & 8 & $\mathbf{C T 1 2}$ & $\mathrm{X}_{1 \mathrm{~T}}$ & $\mathrm{X}_{2 \mathrm{~T}}$ & 2,80 & 8 \\
\hline CT6 & $\mathrm{X}_{1 \mathrm{~T}}$ & 1,40 & 1,40 & 8 & CT13 & $\mathrm{X}_{1 \mathrm{~T}}$ & $\mathrm{X}_{2 \mathrm{~T}}$ & $\mathrm{X}_{3 \mathrm{~T}}$ & 10 \\
\hline CT7 & $\mathrm{X}_{1 \mathrm{~T}}$ & 2,80 & 1,40 & 8 & CT14 & $\mathrm{X}_{1 \mathrm{~T}}$ & $\mathrm{X}_{2 \mathrm{~T}}$ & $\mathrm{X}_{3 \mathrm{~T}}$ & 6 \\
\hline
\end{tabular}

Yêu cầu: Hiệu suất bao là cao nhất và độ hoà tan trong mồi trường đệm phosphat $\mathrm{pH} 6,8$ ở 30 phút không nhỏ hơn $80 \%$.

Khảo sát lớp bao cách ly. Thành phần công thức dịch bao cách ly được trình bày trong bảng 4.

Bảng 4. Thành phần dịch bao cách ly

\begin{tabular}{|c|c|c|}
\hline Thành phân & $\begin{array}{c}\text { Khối } \\
\text { Iượng }(\mathbf{g})\end{array}$ & Vai trò \\
\hline HPMC E6 & 3,00 & Polymer \\
\hline PEG 6000 & Khào sát & Chất hoá dẻo \\
\hline MgO & 0,32 & Chất kiềm \\
\hline Talc & 0,37 & Chống dính \\
\hline TiO2 & 0,25 & Tạo độ đục \\
\hline Tween 80 & 0,06 & Ôn định dịch bao \\
\hline $\begin{array}{c}\text { Hôn hợp EtOH: } \\
\mathrm{H}_{2} \mathrm{O}\end{array}$ & Khảo sát & Dung môi \\
\hline
\end{tabular}

Pha chế dịch bao: hoà tan HPMC E6, PEG6000 và tween 80 trong hỗn hợp cồn: nước được (2). Cho từ từ bột $\mathrm{TiO}_{2}, \mathrm{MgO}$, talc vào (2) khuây đều trên máy khuây từ với tốc độ 200 vòng/phút đến khi đồng nhất, lọc qua rây $125 \mu \mathrm{m}$ thu được dịch bao cách ly. Dịch bao được khuây đều liên tục trong suốt quá trình bao để đảm bảo các chất rắn được phân bố đồng nhất trong hỗn dịch.

Tiển hành bao lớp bao cách ly lên hạt mang hoạt chất bằng phương pháp bồi dần hỗn dịch trên máy sây tầng sôi Mini Glatt với các thông số bao như ở bảng 2. Sau khi hết dịch tiếp tục sấy hạt ở $45^{\circ} \mathrm{C}$ trong 15 phút. Hạt sau khi sây được lấy ra bảo quản ở lọ thuỷ tinh nâu, kín. Khảo sát thành phần công thức dịch bao cách ly gồm:

$Y_{1}$ : tỷ lệ cồn: nước $(1: 1,2: 1,3: 1)$; $Y_{1 T}$ : tỷ lệ cồn : nước tối ưu

$Y_{2}$ : khối lượng PEG $6000(0,3 g ; 0,6 g ; 0,9 g$; 1,2g); Y2т: khối lượng PEG 6000 tối ưu

$$
\mathrm{Y}_{3} \text { : hàm lượng chất khô }(5 \%, 6 \%, 7 \%)
$$

Bảng 5. Thành phần các công thức dịch bao cách ly

\begin{tabular}{|c|c|c|c|c|c|c|c|}
\hline CT & $\mathbf{Y}_{\mathbf{1}}$ & $\mathbf{Y}_{\mathbf{2}}$ & $\mathbf{Y}_{\mathbf{3}}$ & $\mathbf{C T}$ & $\mathbf{Y}_{\mathbf{1}}$ & $\mathbf{Y}_{\mathbf{2}}$ & $\mathbf{Y}_{\mathbf{3}}$ \\
\hline CT15 & $1: 1$ & 0,9 & 6 & $\mathbf{C T 1 9}$ & $\mathrm{Y}_{1 \mathrm{~T}}$ & 0,6 & 6 \\
\hline CT16 & $2: 1$ & 0,9 & 6 & $\mathbf{C T 2 0}$ & $\mathrm{Y}_{1 \mathrm{~T}}$ & 1,2 & 6 \\
\hline CT17 & $3: 1$ & 0,9 & 6 & $\mathbf{C T 2 1}$ & $\mathrm{Y}_{1 \mathrm{~T}}$ & $\mathrm{Y}_{2 \mathrm{~T}}$ & 7 \\
\hline CT18 & $\mathrm{Y}_{1 \mathrm{~T}}$ & 0,3 & 6 & $\mathbf{C T 2 2}$ & $\mathrm{Y}_{1 \mathrm{~T}}$ & $\mathrm{Y}_{2 \mathrm{~T}}$ & 5 \\
\hline
\end{tabular}

Yêu câu: Hiệu suất bao là cao nhất và độ hoà tan trong môi trường đệm phosphat pH 6,8 ở 30 phút không nhỏ hơn $80 \%$.

Khảo sát lớp bao tan trong ruột

Thành phần công thức dịch bao tan trong ruột được trình bày trong bảng 6.

Bảng 6. Thành phần dịch bao tan trong ruột

\begin{tabular}{|c|c|c|}
\hline Thành phân & $\begin{array}{c}\text { Khối } \\
\text { lương(g) }\end{array}$ & Vai trò \\
\hline $\begin{array}{c}\text { Hốn dịch Eudragit }{ }^{\circledR} \\
\text { L30D-55 }\end{array}$ & 80 & $\begin{array}{r}\text { Polymer } \\
\mathrm{r}\end{array}$ \\
\hline & 4 & á dẻo \\
\hline & hảo sát & Chá \\
\hline & & Ön đì \\
\hline Nước & vđ & Dung môi \\
\hline
\end{tabular}

Pha chế dịch bao: Hoà tan hoàn toàn PEG 6000 trong nước. Cho dung dịch này từ từ vào cốc chứa hỗn dịch Eudragit ${ }^{\circledR}$ L30D-55 và khuây đều bằng máy khuấy từ với tốc độ 200 vòng/phút trong 5 phút được (3). Cho từ từ bột
$\mathrm{TiO}_{2}$ vào (3) và khuấy đều bằng máy khuây từ với tốc độ 200 vòng/phút đến khi đồng nhất, lọc qua rây $125 \mu \mathrm{m}$ được dịch bao tan trong ruột. Dich bao được khuấy đều liên tục trong suốt quá trình bao để đảm bảo các chất rắn được phân bố đồng nhất trong hỗn dịch.

Tiến hành bao lớp bao tan trong ruột lên hạt bao lót chất bằng phương pháp bồi dần hốn dịch trên máy sấy tầng sôi Mini Glatt với các thông số bao như ở bảng 2 . Sau khi hết dịch tiếp tục sấy hat ở $45^{\circ} \mathrm{C}$ trong 15 phút. Hạt sau khi sây được lấy ra bảo quản ở lọ thuỷ tinh nâu, kín.

Khảo sát các yếu tố ảnh hưởng đến độ hoà tan của hạt trong môi truòng $\mathrm{HCl} 0,1 \mathrm{~N}$ và môi trường đệm phosphat pH6,8 gồm:

$\mathrm{Z}_{1}$ : khối lượng $\mathrm{TiO}_{2}(0 \mathrm{~g}, 1 \mathrm{~g}, 2 \mathrm{~g}, 3 \mathrm{~g})$. $\mathrm{Z}_{1 \mathrm{~T}}$ : khối lượng $\mathrm{TiO}_{2}$ tối ưu (g) 
$\mathrm{Z}_{2}$ : độ tăng trọng của lớp bao tan trong ruột (20\%, 25\%, 30\%, 35\%, 40\%)

Bảng 7. Thành phần các công thức dịch bao tan trong ruột

\begin{tabular}{|c|c|c|c|c|c|}
\hline CT & $\mathbf{Z}_{\mathbf{1}}$ & $\mathbf{Z}_{\mathbf{2}}$ & $\mathbf{C T}$ & $\mathbf{Z}_{\mathbf{1}}$ & $\mathbf{Z}_{\mathbf{2}}$ \\
\hline $\mathbf{C T 2 3}$ & 0 & 25 & $\mathbf{C T 2 7}$ & $\mathrm{Z}_{1 \mathrm{~T}}$ & 20 \\
\hline CT24 & 1 & 25 & $\mathbf{C T 2 8}$ & $\mathrm{Z}_{1 \mathrm{~T}}$ & 30 \\
\hline CT25 & 2 & 25 & $\mathbf{C T 2 9}$ & $\mathrm{Z}_{1 \mathrm{~T}}$ & 35 \\
\hline CT26 & 3 & 25 & $\mathbf{C T 3 0}$ & $\mathrm{Z}_{1 \mathrm{~T}}$ & 40 \\
\hline
\end{tabular}

Yêu câu: Độ hoà tan của hạt trong môi trường $\mathrm{HCl} 0,1 \mathrm{~N}$ trong 2 giờ thấp nhất và trong môi trường đệm phosphat $\mathrm{pH} 6,8$ trong 30 phút không nhỏ hơn 80\%.
Hạt sau khi bao lớp bao tan trong ruột sẽ được tiến hành kiểm nghiệm bán thành phẩm với các chỉ tiêu như sau: tỷ trọng biểu kiến, độ mài mòn, độ ẩm, tốc độ chảy.

Hạt sẽ được đóng nang vào cõ̃ nang số 1 và kiểm nghiệm viên nang chứa esomeprazol $20 \mathrm{mg}$ dạng hat bao tan trong ruột theo tiêu chuẩn của USP43 ${ }^{[\dot{ }]}$.

\section{KẾT QUẢ NGHIÊN CỨU}

Khảo sát lớp bao mang hoạt chất. Kết quả khảo sát thành phần lớp bao mang hoạt chất được trình bày ở bảng 8 .

Bảng 8. Kết quả khảo sát thành phần lớp bao mang hoat chất

\begin{tabular}{|c|c|c|c|c|c|c|c|c|c|c|c|c|c|}
\hline CT & $\mathbf{X}_{\mathbf{1}}$ & $\mathbf{X}_{\mathbf{2}}$ & $\mathbf{X}_{\mathbf{3}}$ & $\mathbf{X}_{\mathbf{4}}$ & $\mathbf{a}$ & $\mathbf{b}$ & $\mathbf{C T}$ & $\mathbf{X}_{\mathbf{1}}$ & $\mathbf{X}_{\mathbf{2}}$ & $\mathbf{X}_{\mathbf{3}}$ & $\mathbf{X}_{\mathbf{4}}$ & $\mathbf{a}$ & $\mathbf{b}$ \\
\hline CT1 & $1: 1$ & 2,10 & 1,40 & 8 & - & - & $\mathbf{C T}$ & $3: 1$ & 3,50 & 1,40 & 8 & - & - \\
\hline CT2 & $2: 1$ & 2,10 & 1,40 & 8 & 71 & 94,7 & CT9 & $3: 1$ & 2,10 & 0,35 & 8 & - & - \\
\hline CT3 & $3: 1$ & 2,10 & 1,40 & 8 & 85 & 94,2 & CT10 & $3: 1$ & 2,10 & 0,70 & 8 & 71 & 92,1 \\
\hline CT4 & $4: 1$ & 2,10 & 1,40 & 8 & 79 & 93,8 & CT11 & $3: 1$ & 2,10 & 2,10 & 8 & 86 & 94,8 \\
\hline CT5 & $3: 1$ & 0,70 & 1,40 & 8 & 65 & 91,1 & CT12 & $3: 1$ & 2,10 & 2,80 & 8 & 80 & 95,3 \\
\hline CT6 & $3: 1$ & 1,40 & 1,40 & 8 & 71 & 92,8 & CT13 & $3: 1$ & 2,10 & 2,10 & 10 & 68 & 93,9 \\
\hline CT7 & $3: 1$ & 2,80 & 1,40 & 8 & 84 & 93,8 & CT14 & $3: 1$ & 2,10 & 2,10 & 6 & 83 & 95,0 \\
\hline
\end{tabular}

Với -: không ghi nhân được kết quả; $X_{1}$ : tỷ lê cồn: nước; $X_{2}$ : khối lượng PEG $6000(\mathrm{~g}) ; X_{3}$ : khối lượng talc $(\mathrm{g})$; $\mathrm{X}_{4}$ : hàm lượng chất khô $(\%)$; $\mathrm{a}$ : hiệu suất bao (\%); b: độ hoà tan của hạt trong đệm phosphat $\mathrm{pH}$ 6,8 trong 30 phút (\%).

Nhân xét: Về ảnh hưởng của tỷ lệ cồn: nước ở các CT1, CT2, CT3, CT4 cho thây CT3 cho hiệu suất bao cao nhất (85\%), đồng thời độ hoà tan đạt $(94,2 \%>80 \%)$. Vậy chọn tỷ lệ cồn: nước = 3:1. Ở CT3, CT5, CT6, CT7, СT8 cho thấy CT3 cho hiệu suất cao nhất $(85 \%)$, độ hoà tan đạt
$(94,2 \%>80 \%)$, nên chọ khối lượng PEG 6000 bằng $2,10 \mathrm{~g}$ để bào chế. Ở CT3, CT9, CT10, CT11, CT12 thì CT11 có hiêuu suất bao cao nhất $(86 \%)$, độ hoà $\tan$ đạt $(94,8 \%>80 \%)$, nên khối lượng talc được chọn là 2,10g. Ở CT11, CT13, CT14 cho thấy CT11 có hiệu suất bao cao nhất $(86 \%)$, đồng thời độ hoà tan đạt $(94,8 \%>$ $80 \%$ ), nên hàm lượng chất khô được chọn là $8 \%$.

Khảo sát lớp bao cách ly. Kểt quả khảo sát thành phần dịch bao cách ly được trình bày trong bảng 9

Bảng 9. Kết quả khảo sát thành phần công thức dịch bao cách ly

\begin{tabular}{|c|c|c|c|c|c|c|c|c|c|c|c|}
\hline CT & $\mathbf{Y}_{\mathbf{1}}$ & $\mathbf{Y}_{\mathbf{2}}$ & $\mathbf{Y}_{\mathbf{3}}$ & $\mathbf{a}$ & $\mathbf{b}$ & $\mathbf{C T}$ & $\mathbf{Y}_{\mathbf{1}}$ & $\mathbf{Y}_{\mathbf{2}}$ & $\mathbf{Y} \mathbf{3}$ & $\mathbf{a}$ & $\mathbf{b}$ \\
\hline CT15 & $1: 1$ & 0,9 & 6 & 72 & 86,2 & $\mathbf{C T 1 9}$ & $3: 1$ & 0,6 & 6 & 79 & 85,8 \\
\hline CT16 & $2: 1$ & 0,9 & 6 & 76 & 85,7 & $\mathbf{C T 2 0}$ & $3: 1$ & 1,2 & 6 & 87 & 87,2 \\
\hline CT17 & $3: 1$ & 0,9 & 6 & 83 & 86,7 & $\mathbf{C T 2 1}$ & $3: 1$ & 1,2 & 7 & 76 & 83,3 \\
\hline CT18 & $3: 1$ & 0,3 & 6 & 75 & 85,2 & $\mathbf{C T 2 2}$ & $3: 1$ & 1,2 & 5 & 85 & 86,8 \\
\hline
\end{tabular}

Với $Y_{1}$ : tỷ lê cồn: nước; $Y_{2}$ : khối lượng PEG $6000(\mathrm{~g}) ; Y_{3}$ : hàm lượng chất khô $(\%)$; a: hiệu suất bao (\%); b: độ hoà tan của hạt trong đệm phosphat $\mathrm{pH} 6,8$ trong 30 phút (\%)

Nhận xét: Từ kết quả CT15, CT16, CT17 cho thấy CT17 cho hiệu suất bao cao nhất $(83 \%)$, đồng thời độ hoà tan đạt $(86,7 \%>80 \%)$, vì thế tỷ lệ cồn: nước được chọn là $3: 1$. Ở CT17, CT18, CT19, CT20 cho thấy CT20 cho hiệu suất bao cao nhất $(87 \%)$, độ hoà tan đạt $(87,2 \%>$ 80\%), nên khối lượng PEG 6000 được chọn là 1,2g. Ở CT20, CT21, CT22 cho hiệu suất bao cao nhất $(87 \%)$ và độ hoà tan đạt $(87,2 \%>80 \%)$, nên hàm lượng chất khô được chọn là $6 \%$.

Khảo sát lớp bao tan trong ruột. Kết quả khảo sát thành phần công thức lớp bao tan trong ruột trình bày ở bảng 10 .

Bảng 10. Kết quả khảo sát thành phần công thức dịch bao tan trong ruột

\begin{tabular}{|c|c|c|c|c|c|c|c|c|c|}
\hline $\mathbf{C T}$ & $\mathbf{Z}_{\mathbf{1}}$ & $\mathbf{Z}_{\mathbf{2}}$ & $\mathbf{C}$ & $\mathbf{b}$ & $\mathbf{C T}$ & $\mathbf{Z} \mathbf{1}$ & $\mathbf{Z} \mathbf{2}$ & $\mathbf{C}$ & $\mathbf{b}$ \\
\hline CT23 & 0 & 25 & 16 & $\mathbf{7 1 , 2}$ & $\mathbf{C T 2 7}$ & 1 & 20 & 19,2 & 91,1 \\
\hline CT24 & 1 & 25 & 11,4 & 82,3 & $\mathbf{C T 2 8}$ & 1 & 30 & 8,7 & 81,8 \\
\hline CT25 & 2 & 25 & 15,8 & 74,2 & CT29 & 1 & 35 & 6,7 & 79,2 \\
\hline CT26 & 3 & 25 & 18,2 & 75,5 & CT30 & 1 & 40 & 5,2 & 73,9 \\
\hline
\end{tabular}


Với $Z_{1}$ : khối lượng $\mathrm{TiO}_{2}$ trong công thức $(\mathrm{g})$; $Z_{2}$ : độ tăng trọng của lớp bao tan trong ruột (\%);c: độ hoà tan của hạt trong môi trường acid $\mathrm{HCl} 0,1 \mathrm{~N}$ trong 2 giờ $(\%) ;$ b: độ hoà tan của hạt trong đêm phosphat pH 6,8 trong 30 phút (\%)

Nhận xét: Từ kết quả CT23, CT24, CT25, CT26 cho thấy CT24 cho độ hoà tan của hạt trong môi trường acid $\mathrm{HCl} 0,1 \mathrm{~N}$ thấp nhất và độ hoà tan của hat trong môi trường đệm phosphat pH 6,8 cao nhất, nên chọn khối lượng $\mathrm{TiO}_{2}$ là $1 \mathrm{~g}$. Ở CT24, CT27, CT28, CT29, CT30 cho thây CT28 cho độ hoà tan của hạt trong môi trường acid $\mathrm{HCl} 0,1 \mathrm{~N}$ đạt $(8,7 \%<10 \%)$ và độ hoà tan của hạt trong môi trường đệm phosphat pH 6,8 đạt $(81,8 \%>80 \%)$. Do đó, chọn độ tăng trọng của lớp bao tan trong ruột là $30 \%$.

Thành phân công thức viên nang chứa esomeprazol 20mg dạng hạt bao tan trong ruột được trình bày ở bảng 11 .

Bảng 11. Thành phần công thức viên nang chứa esomeprazol $20 \mathrm{mg}$ dạng hạt bao tan trong ruột

\begin{tabular}{|c|c|c|}
\hline & Thành phân & $\begin{array}{c}\text { Khối } \\
\text { lượng(g) }\end{array}$ \\
\hline \multirow{7}{*}{$\begin{array}{c}\text { Lớp bao } \\
\text { mang } \\
\text { hoạt chất }\end{array}$} & Esomeprazol magnesi & 3,19 \\
\hline & Sugarsphere $600-710 \mu \mathrm{m}$ & 30 \\
\hline & $\mathrm{MgO}$ & 3,31 \\
\hline & HPMC E6 & 3,00 \\
\hline & PEG 6000 & 1,81 \\
\hline & Talc & 1,81 \\
\hline & Tween 80 & 0,15 \\
\hline \multirow{6}{*}{$\begin{array}{c}\text { Lớp bao } \\
\text { cách ly }\end{array}$} & HPMC E6 & 2,61 \\
\hline & PEG 6000 & 1,04 \\
\hline & $\mathrm{MgO}$ & 0,28 \\
\hline & Talc & 0,32 \\
\hline & $\mathrm{TiO2}$ & 0,22 \\
\hline & Tween 80 & 0,05 \\
\hline \multirow{4}{*}{$\begin{array}{c}\text { Lớp bao } \\
\text { tan trong } \\
\text { ruột }\end{array}$} & Eudragit ${ }^{\circledR}$ L30D-55 & 12,86 \\
\hline & PEG 6000 & 1,28 \\
\hline & $\mathrm{TiO}_{2}$ & 0,54 \\
\hline & Tween & 0,75 \\
\hline
\end{tabular}

Viên nang chứa esomeprazol 20mg dang hat bao tan trong ruột đều đạt các chỉ tiêu kiểm nghiệm như cảm quan, độ đồng đều khối lượng, độ hoà tan, định tính, định lượng theo tiêu chuẩn USP43.

\section{BÀN LUẬN}

Các dung môi thường được sử dụng trong quá trình bồi dần bằng hỗn địch thường là aceton, methanol, isopropanol hoặc sự kết hợp các dung môi. Tuy nhiên, để giảm nguy cơ hạt bị dính trong quá trình bồi, sử dụng dung môi có chi phí thấp và hạn chế nguy cơ cháy nổ, chúng tôi quyết định chọn hỗn hợp ethanol và nước. Nước là dung môi hoà tan tốt các tá dược HPMC
E6, PEG 6000, Tween 80 lại có chi phí thấp. Tuy nhiên, một số nhược điểm của nước là tạo dịch nhớt với HPMC E6, PEG 6000 và tạo bọt bền khi có mặt cùng với Tween 80 , khả năng hoà tan hoạt chất kém, khả năng bay hơi kém. Ethanol có ưu điểm là dung môi hoà tan tốt các polymer và tạo dịch có độ nhớt thấp, phá bọt và hoà tan hoạt chất tốt hơn so với nước nhưng đắt tiền, dễ bay hơi, dễ cháy nổ. Chính vì vậy, ethanol được sử dụng kết hợp với nước theo các tỷ lệ để làm dung môi pha dịch bao mang hoạt chất và dịch bao cách ly[2].

Hiệu suất bao thay đổi có thể giải thích là do tăng tỷ lệ tá dược dính thì số cầu nối giữa các chuối phẩn tử polymer để kết nối các tiểu phân tăng lên, do đó giúp chất rắn bám trên bề mă̆t nhân tốt hơn. Nhưng nếu tiếp tục tăng tỷ lệ tá dược dính đến một mức nào đó số cầu nối đã đủ lượng chất rắn bám vào bề mặt hạt nên hiệu suất không tăng thêm nữa. Ngoài ra, nếu tỷ lệ tá dược dính tăng quá cao làm tăng độ nhớt dịch bao, giảm tốc độ bay hơi dẫn đến các hạt tiếp xúc, dính vào nhau gây hiện tượng dính chùm, đóng cục và bám vào thiết bị (CT8). PEG 6000 đóng vai trò vừa là tá dược dính vừa là chất hoá dẻo, là polymer tan rất tốt trong nước giúp giải phóng nhanh hoạt chất. Khi tăng tỷ lệ PEG 6000 thì độ hoà tan tăng, điều này phù hợp với nghiên cứu của Yongmei Xie cho rằng PEG 6000 có khả năng tăng cường độ tan của muối esomeprazo[[5]. Nhưng khi tăng PEG 6000 lên nữa thì độ hoà tan lại giảm đi một ít, điều này có thể giải thích là do khi đo hoà tan trong môi trường đệm pH 6,8 các hạt có tính bám dính cao nên sẽ tập trung và kết tụ thành khối nên giữ lại hoạt chất bên trong lâu hơn là công thức có lượng tá dược dính thấp.

Khi sử dụng lượng talc quá thấp thì không đủ khả năng chống kết dính các hạt trong khi bao, gây nên hiện tượng dính chùm và đóng cục. Nếu tăng lượng talc các giọt dịch bao bám lên hạt tạo bề mặt gồ ghề hơn, tăng diện tích tiếp xúc với khối khí nên hạt nhanh khô. Đồng thời talc đóng vai trò cầu nối trung gian gắn kết các chuỗi phân tử polymer, làm chất rắn bám tốt trên bề mặt hạt, nhưng lượng talc tăng đến một mức nào đó trong công thức thì tạo đủ số lượng cầu nối nên nếu tăng lên thì hiệu suất lại giảm.

Hàm lượng chất khô là một yếu tố chính ảnh hưởng đến quá trình bao. Khi hàm lượng chất khô lớn tức dịch bao có độ đậm đặc cao, súng phun có thể bị nghẹt không những vậy, lượng polymer trong dịch cũng tăng lên làm tăng độ nhớt của dịch bao, gây hiện tượng dính hạt khi phun ${ }^{[2]}$. 
Ngoài thành phần công thức, độ tăng trọng của lớp bao tan trong ruột là một trong những yếu tố quan trọng nhất giúp hạt kháng lại môi trường acid. Độ tăng trọng lớp bao càng tăng, khả năng kháng acid càng tốt nên độ giải phóng hoạt chát hoạt chất trong môi trường acid $\mathrm{HCl}$ $0,1 \mathrm{~N}$ giảm. Tuy nhiên khả năng giải phóng hoạt chất trong môi trường đệm $\mathrm{pH}$ 6,8 cũng giảm theo. Do đó, độ dày lớp bao tan trong ruột 30\% là đạt yêu cầu.

\section{KẾT LUÂNN}

Đã bào chế được viên nang chứa esomeprazol $20 \mathrm{mg}$ dang hạt bao tan trong ruột đạt các tiêu chuẩn kiểm nghiệm của USP43.
TÀI LIẸU THAM KHẢO

1. Chemical Medicines Monographs 3 Expert Committee (2016), "Esomeprazole Magnesium Delayed-Release Capsules", pp.1-4.

2. Shu-Ling Kan et al (2014), "Preparation and in vitro/in vivo evaluation of esomeprazole magnesium modified release", Informa healthcare,pp.1-8.

3. Singh Deep Hussan et al (2012), "A review on recent advances of enteric coating", IOSR Journal of Pharmacy, vol 2, pp.05-11.

4. USP43-NF38 (2019), The United States Pharmacopedial Convention.

5. Yongmei Xie, Ping Xie (2008), "Preparation of esomeprazole zinc solid dispersion and study on its pharmacokinetics", pp.53-57.

\section{SỐ LƯợNG ỐNG TỦY CHÂN RĂNG CỦA RĂNG CỐI LỚN THỨ NHẤT HÀM DƯỚI TRÊN CONEBEAM CT TRONG ĐIỀU TRI NHA KHOA}

\section{TÓM TẮT}

Mục tiêu: Đây là nghiên cứu khảo sát trên phim ConeBeam $\mathrm{CT}$ nhằm xác định số ống tủy của răng cối lớn (RCL) thứ nhất hàm dưới ở người Việt Nam. Đối tương và phương pháp: Nghiên cứu được thực hiện trên 166 bệnh nhân chụp phim CBCT theo chỉ định của bác sĩ tai Trung tâm CT nha khoa Nguyễn Trãi, Thành Phố Hồ Chí Minh, trong thời gian nghiên cứu từ tháng 10/2015 đến tháng 6/2016. Phim CBCT được chụp bằng máy chụp phim Picasso Trio (Ewoo Vatech, Korea) với các điều kiện và tư thế chuẩn của bệnh nhân cho chụp phim. Hình ảnh $\mathrm{CB} C \mathrm{~N}$ thu thập từ trung tâm CT đạt tiêu chuẩn chọn mẫu được quạn sát trên máy tính màn hình phẳng 14 inches, độ phân giải 1366 x 768 pixel với phần mềm EzImplant CD viewer. Ghi nhận vị trí răng (răng 36 và răng 46), khảo sát số lượng ống tủy của răng cối lớn thứ nhất hàm dưới trển hình ảnh $\mathrm{CBCT}$ : trong mặt phẳng ngang (Axial), di chuyển các lát cắt trên thiết diện ngang của ống tủy từ sàn tủy đến chóp. Quan sát theo thiết diện ngang ở những mức sau: miệng ống tủy, phần ba giữa chân răng, phần ba chóp chân răng. Quan sát ống tủy của từng chân răng của răng cối lớn thứ nhất hàm dưới theo ba măt phẳng. Xác đinh số lượng ống tủy. Kết quả: Tỉ lệ RCL thứ nhất hàm dưới có hai ông tủy, ba ống tủy, bốn ống tủy, lần lượt là $4,5 \%, 66,8 \%$ và $28,9 \%$. Sự phân bố này khác biệt không có ý nghĩa theo giới tính và theo vị trí. Kết luận: Tî lệ RCL thứ nhất hàm dưới trên CONEBEAM CTं có ba ống tủy

${ }^{1}$ Đại học Y Dước TP.HCM

Chịu trách nhiệm chính: Huỳnh Kim Khang

Email: kimkhanghuynh@yahoo.com

Ngày nhận bài: 8/3/2021

Ngày phản biện khoa học: 4/4/2021

Ngày duyệt bài: 2/5/2021
Huỳnh Kim Khang ${ }^{1}$, Phạm Văn Khoa ${ }^{1}$

chiếm đa số

Tư khóa: Ống tủy chân răng, răng cối lớn thứ nhất hàm dưới, ConeBeam CT.

Viết tắt: RCL1: răng cối lớn thứ nhất; $\mathrm{CBCT}$ : ConeBeam CT

\section{SUMMARY}

THE NUMBER OF THE ROOT CANALS OF THE FIRST LOWER MOLARS ON CONEBEAM CT IN DENTAL TREATMENT

Objectives: The aim of the study is to determine the number of the root canals of the first lower molars in Vietnamese on ConeBeam CT. Subjects and methods: The study was conducted on 166 patients who had exposured using CBCT indicated by dentists in Nguyen Trai Dental CT Central, HoChiMinh City, from October 2015 to June 2016. The CBCT digital images were captures using Picasso Trio (Ewoo Vatech, Korea) with the standard conditions and postures of patients. CBCT digital images were displayed on the 14 inches flat monitor, at 1366 × 768 pixel resolution with EzImplant CD viewer software. The positions of the first lower molars were recorded. The number of root canals of the first lower molars was examined by moving cross-sectional slices from the pulpal floor to the apex. The orifices, middle thirds, apical thirds of the canals of the first lower molar was observed and the root canals of each root of the first lower molars were observed in three planes Results: The prevalences of two, three, four root canals of the first lower molars were $4.5 \%$, $66.8 \%$ and $28.9 \%$ respectively. There were not significant differences about sex and positions. Conclusion:The prevalences of three root canals of the first lower molars accounts for the largest portion.

Key words: Root canals, first lower molar, ConeBeam CT. 